\title{
Cone-Beam Computed Tomography
}

National Cancer Institute

\section{Source}

National Cancer Institute. Cone-Beam Computed Tomography. NCI Thesaurus. Code C71695.

A method of computed tomography that utilizes a cone shaped X-ray beam that captures a full volume of image as the X-ray source and detector simultaneously move in a single rotation around a stabilized target. 